\title{
Magnetic field evolves to gravity field part: 3 Electromagnets
}

\author{
John C. Hodge ${ }^{1 *}$ \\ ${ }^{1}$ Retired, 477 Mincey Rd., Franklin, NC, 28734
}

\begin{abstract}
The STOE suggests gravity emerges from magnetic effects of hods in matter structures. The Scalar Theory of Everything (STOE) proposes gravity is an asymmetry (only attraction) in the magnetic field of particles. Differences between the poles of permanent magnets are absent in electromagnets. Therefore, the magnetic field of particles is similar to the magnetic field of permanent magnets. Models suggesting revolving charges causes particle magnetic fields are rejected because of increased complexity and reduced inclusion.
\end{abstract}

keywords: magnet, gravity, electromagnets

\section{INTRODUCTION}

Experiments rejected the Biot-Savart Law (Hodge 2018b). The Scalar Theory of Everything (STOE) suggests two different types of magnetic field in Maxwell's equations (Hodge 2018d). One type is from permanent magnets; the other is from electric current (electromagnets). The STOE suggests the hods, photons, and particles are modeled by assembles of permanent disc magnets (Hodge 2016a). Gravity may be the sum of the poles at the side of photons or the photons comprising particles (Hodge 2019a).

Table 1 (corrected) shows the North Pole of permanent magnets has statistically larger force exerted $W$ vs distance $d^{-2}$ slope than the South Pole while the $W$ relation with $d^{-1}$ is nearly identical (Hodge 2019a). This would speculate the North Pole dominates at the side of the photon (bar) configuration as suggested in Hodge (2012).

This Paper examines electromagnets as a cause of the asymmetry in gravity. The description of the experiments is in section 2. The magnetic field effect on iron filings is show in section 3. The Discussion and Conclusion are in section 4.

*E-mail: jchodge@frontier.com 
Table 1: Data from Hodge (2019a).

\begin{tabular}{|c|c|l|c|l|}
\hline \hline & \multicolumn{2}{|c|}{ South-South } & \multicolumn{2}{|c|}{ North-North } \\
\hline run & No. & $W$ & No. & $W$ \\
No. & pts. & $(\mathrm{gw})$ & pts & $(\mathrm{gw})$ \\
\hline Zone 1 & 14 & $(0.70 * 1000 / d-21) \pm 5$ & 13 & $(0.7 * 1000 / d-13) \pm 2.7$ \\
Zone 2 & 15 & $\left(4.35 * 1000 / d^{2}-0.74\right) \pm 0.17$ & 13 & $\left(6.1 * 1000 / d^{2}-1.8\right) \pm 0.8$ \\
Zone 3 & 5 & $75.7 e^{-0.09 d} \pm 0.6$ & 7 & $99.4 e^{-0.1 d} \pm 1.5$ \\
\hline
\end{tabular}

\section{THE EXPERIMENT 1}

As in Hodge (2019a), electromagnets were glued to the wood supports. The scale (AWS-100, Digital scale) measures gram weight $W(\mathrm{gw})$ and has a tolerance of \pm 0.02 gw. A calibration 20 gram mass weighed 19.99 gw. By repeatedly assembling a measurement setup, the scale read $\pm 0.4 \mathrm{gw}$ for small forces. This is attributed to the tolerance of the wood support being poor for small forces. All measurement are $\pm 10 \%$ unless otherwise stated. The distance between the top ends of the iron core $d_{f e}$ and the distance between the top ends of the coils $d_{\text {air }}$ are measured.

Table 2: Linear regression of the data of the Zones of the electromagnet with an iron core.

\begin{tabular}{|c|c|c|c|c|}
\hline & \multicolumn{2}{|r|}{ South-South } & \multicolumn{2}{|r|}{ North-North } \\
\hline $\begin{array}{l}\text { run } \\
\text { No. }\end{array}$ & $\begin{array}{l}\text { No. } \\
\text { pts. }\end{array}$ & $\begin{array}{l}W \\
(\mathrm{gw})\end{array}$ & $\begin{array}{l}\text { No. } \\
\text { pts }\end{array}$ & $\begin{array}{l}W \\
(\mathrm{gw})\end{array}$ \\
\hline Zone 1 & 4 & $\left(0.008 * 1000 / d_{f e}+2\right) \pm .03$ & 4 & $\left(0.008 * 1000 / d_{f e}+2.07\right) \pm .02$ \\
\hline Zone 2 & 12 & $\left(0.73 * 1000 / d_{f e}^{2}+0.11\right) \pm 0.02$ & 12 & $\left(0.69 * 1000 / d_{f e}^{2}+0.41\right) \pm 0.03$ \\
\hline Zone 3 & 17 & $5.24 e^{-0.06 d_{f e}} \pm 0.02$ & 17 & $4.96 e^{-0.05 d_{f e}} \pm 0.4$ \\
\hline
\end{tabular}

Table 3: Linear regression of the data of the Zones of the electromagnet comparing the North-North with an air core distance $d_{\text {air }}$ versus the iron core distance $d_{f e}$.

\begin{tabular}{|c|c|l|c|l|}
\hline \hline & \multicolumn{1}{|c|}{ Air core } & \multicolumn{2}{|c|}{ Iron core } \\
\hline run & No. & $W$ & No. & $W$ \\
No. & pts. & $(\mathrm{gw})$ & $\mathrm{pts}$ & $(\mathrm{gw})$ \\
\hline Zone 1 & 4 & $\left(0.02 * 1000 / d_{\text {air }}+2\right) \pm .05$ & 4 & $\left(0.008 * 1000 / d_{f e}+2.07\right) \pm .02$ \\
Zone 2 & 12 & $\left(0.72 * 1000 / d_{\text {air }}^{2}+0.05\right) \pm 0.02$ & 12 & $\left(0.69 * 1000 / d_{f e}^{2}+0.0 .41\right) \pm 0.03$ \\
Zone 3 & 17 & $4.00 e^{-0.05 d_{\text {air }}} \pm 0.06$ & 17 & $4.96 e^{-0.05 d_{f e} \pm 0.4}$ \\
\hline
\end{tabular}

Tables 1 through 3 lists the linear regression equations with one standard deviation uncertainty. 


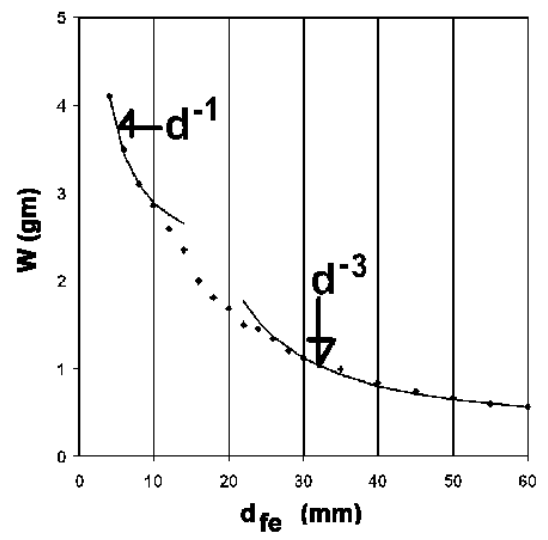

Figure 1: Plot of the $W$ versus $d_{f e}$.

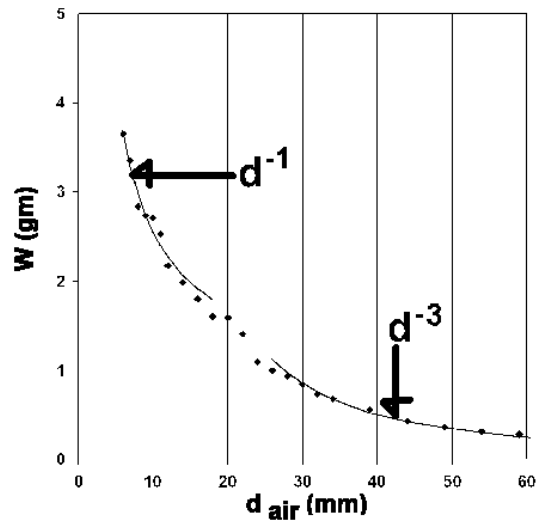

Figure 2: Plot of the $W$ versus $d_{\text {air }}$.

\section{EXPERIMENT 2}

The magnetic fields of various configurations of permanent magnets and electromagnets were examined by use of iron filings.

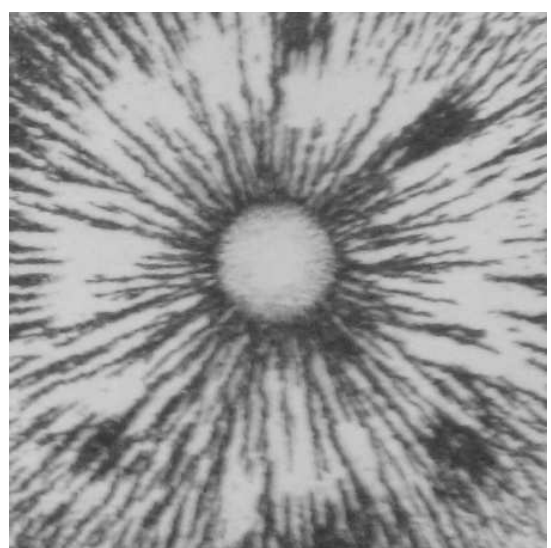

Figure 3: Photo of iron filings of permanent magnets with the axis perpendicular to the plane for the South Pole.

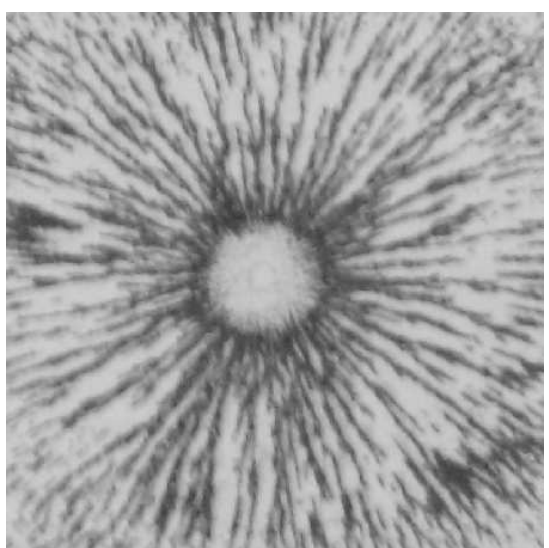

Figure 4: Photo of iron filings of permanent magnets with the axis perpendicular to the plane for the North Pole.. 


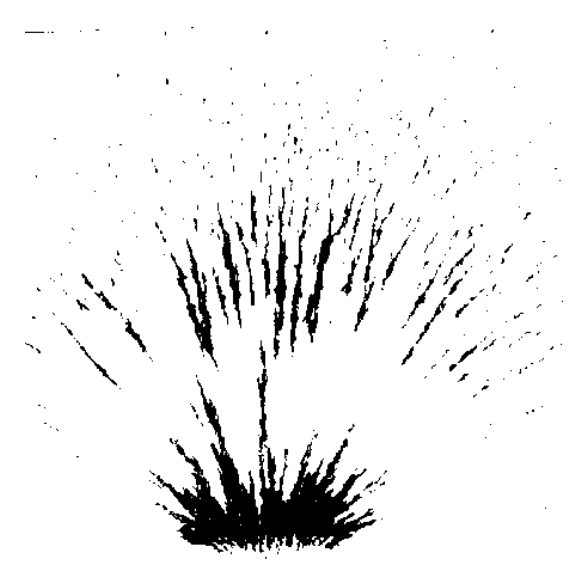

Figure 5: Photo of iron filings of permanent magnets with the axis in the plane for the South Pole.

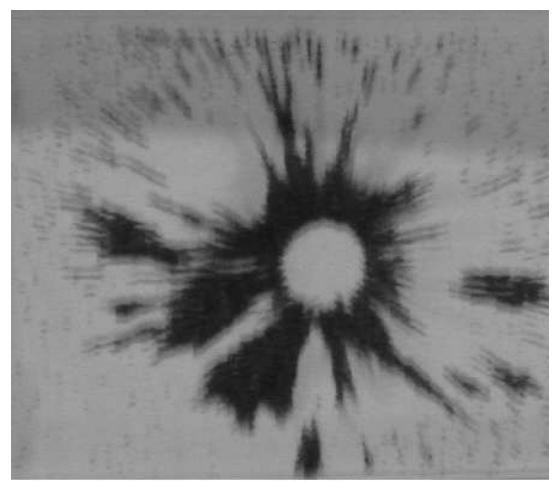

Figure 7: Photo of iron filings of iron core electromagnets with the axis perpendicular to the plane for the South Pole.

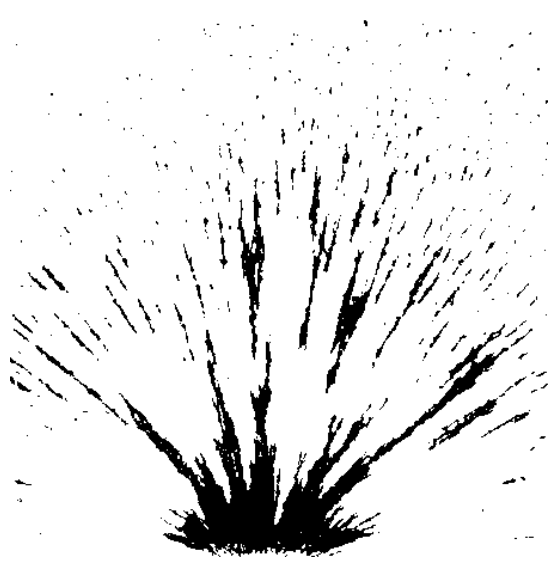

Figure 6: Photo of iron filings of permanent magnets with the axis in the plane for the North Pole.

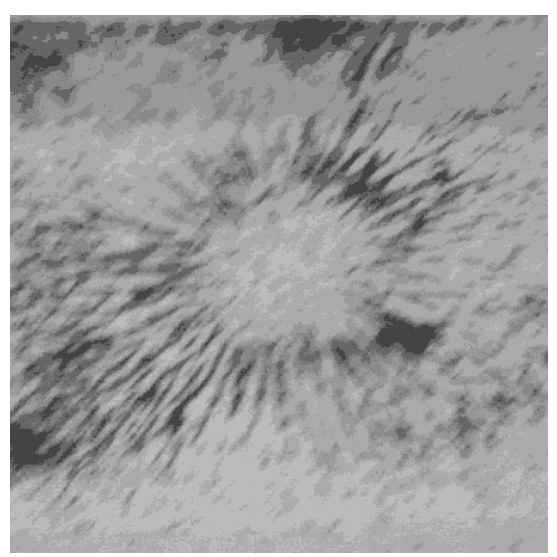

Figure 8: Photo of iron filings of iron core electromagnets with the axis perpendicular to the plane for the North Pole. 


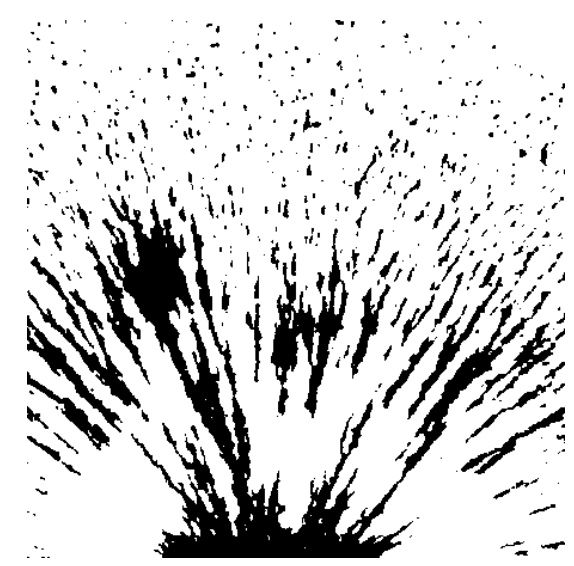

Figure 9: Photo of iron filings of iron core electromagnets with the axis in the plane for the South Pole.

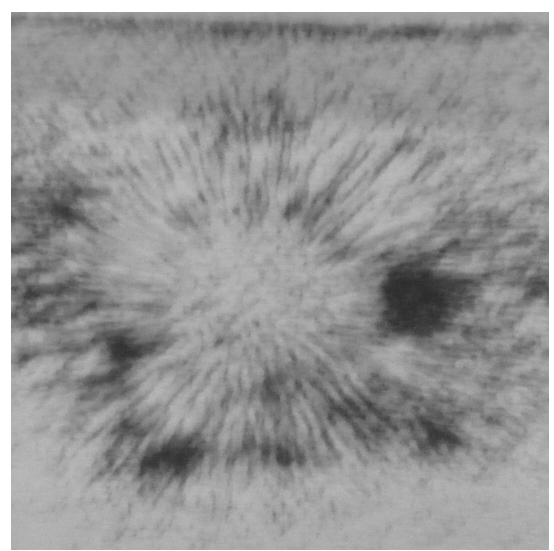

Figure 11: Photo of iron filings of air core electromagnets with the axis perpendicular to the plane for the South Pole.

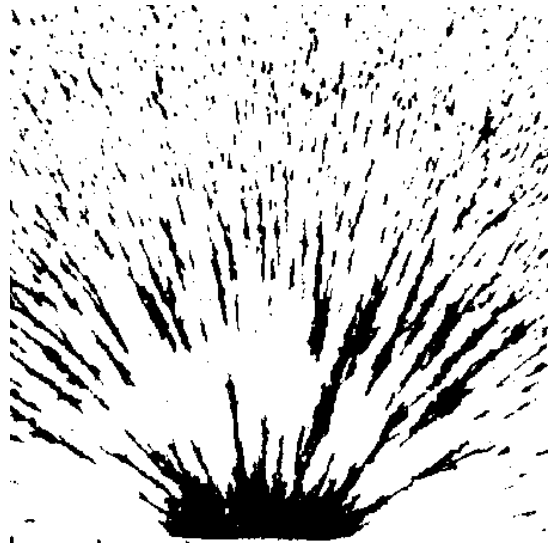

Figure 10: Photo of iron filings of iron core electromagnets with the axis in the plane for the North Pole.

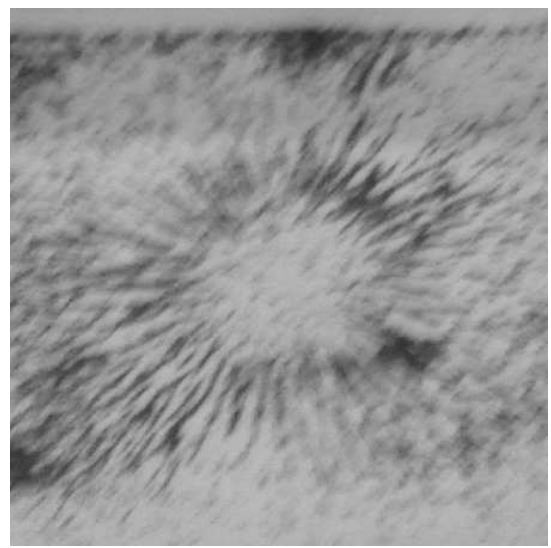

Figure 12: Photo of iron filings of air core electromagnets with the axis perpendicular to the plane for the North Pole. 


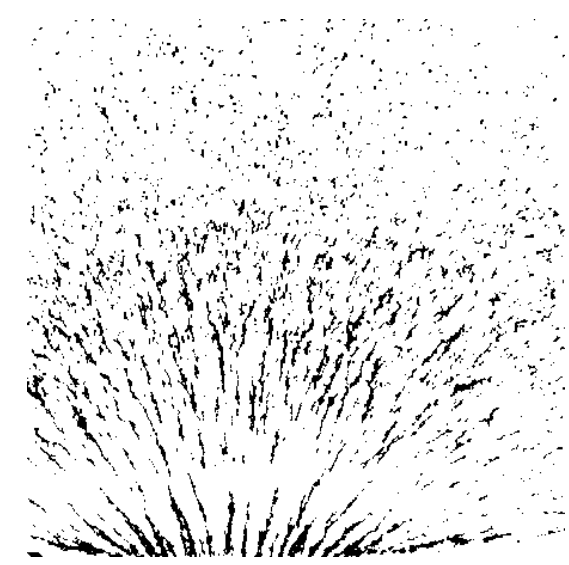

Figure 13: Photo of iron filings of air core electromagnets with the axis in the plane for the South Pole.

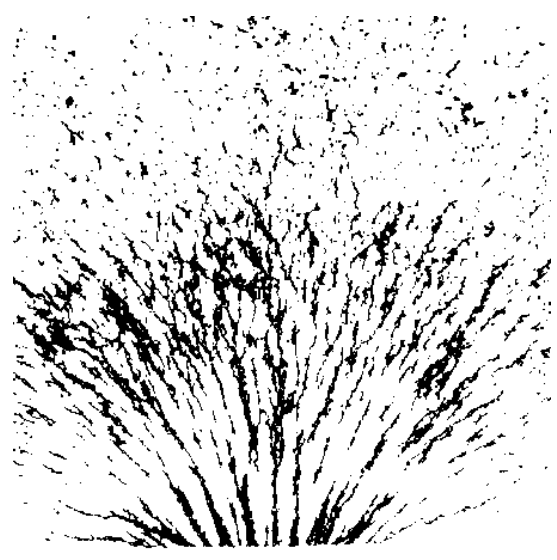

Figure 14: Photo of iron filings of air core electromagnets with the axis in the plane for the North Pole.

\section{DISCUSSION AND CONCLUSION}

The magnetic field of permanent magnets show a difference in force $W$ exerted in Zone 2 (large $d$ ) (Tables 1, Fig. 3 through 6 ). The fields on both air core and iron core electromagnets show little difference (Table 2, Table 3, and Figs. 7 Through 14).

The number of dendrites at given radius from the center is less for the South Pole (56) than the North Pole (64) of permanent magnets. The number of dendrites at given radius from the center is approximately the same for the South Pole and North Pole of both iron core and air core electromagnets. Another difference is that the field from permanent magnets is more focused outward in analogy to a fluid flowing outward from a pipe (by the method of conjugate functions using the transformation $w=z+\mathrm{e}^{z}$ ). The field from electromagnets is more rounded. That is the edges of the field are caused by the edge wire current flow.

Gravity is an asymmetry effect (only attraction) of particles. The STOE proposes gravity is an asymmetry in the magnetic field of particles. Differences between the poles of permanent magnets are absent in electromagnets. Therefore, the magnetic field of particles is similar to the magnetic field of permanent magnets. Models suggesting revolving charges cause particle magnetic fields are rejected because of increased complexity and reduced inclusion.

\section{References}

Hodge, J.C., 2012, Photon diffraction and interference, IntellectualArchive, Vol.1, No. 3, P. 20,. http://intellectualarchive.com/?link=item\&id=597 


\section{REFERENCES}

Hodge, J.C., 2016a, Structure and spin of the neutrino, electron, and positron, IntellectualArchive, Vol.5, No. 5, P. 1,. http://intellectualarchive.com/?link=item\&id=1694

Hodge, J.C., 2016b, STOE emergence, http://intellectualarchive.com/?link=item\&id $=1757$

Hodge, J.C., 2018a, STOE electric charge, IntellectualArchive, Vol.7, No. 2, P 1. 1 , http://intellectualarchive.com/?link=item\&id=1917

Hodge, J.C., 2018b, Magnetostatics relation to gravity with experiment that rejects Biot-Savart Law, IntellectualArchive, Vol.7, No. 3, P. 1,. http://intellectualarchive.com/?link=item\&id=1945

Hodge, J.C., 2018c, Another experiment rejects Ampere's Law and supports the STOE model, IntellectualArchive, Vol.7, No. 4, P. 6,. http://intellectualarchive.com/?link=item\&id=1956

Hodge, J.C., 2018d, Two different types of magnetic field, IntellectualArchive, Vol.4, No. 4, P. 1,. http://intellectualarchive.com/?link=item\&id=1964

Hodge, J.C., 2018e, STOE replaces relativity and quantum mechanics, (ISBN 978-613-9-91465-4, (LAP LAMBERT Acedemic publishing, Mauritius, available through Amazon.com).

Hodge, J.C., 2019a, Magnetic field evolves to gravity field part 1: Repulsion, IntellectualArchive, Vol.8, No. 3, P. 17,. http://intellectualarchive.com/?link=item\&id=2164

Hodge, J.C., 2019b, Magnetic field evolves to gravity field part 1: particles, IntellectualArchive, Vol.8, No. 3, P. 30,. http://intellectualarchive.com/?link $=$ item\&id $=2170$ 